\title{
Autonomous surface vessel for search and rescue operation
}

\author{
Hasmah Mansor ${ }^{1}$, Muhammad Haziq Norhisam² ${ }^{2}$ Zulkifli Zainal Abidin ${ }^{3}$, Teddy Surya Gunawan ${ }^{4}$ \\ ${ }^{1,2,4}$ Department of Electrical and Computer Engineering, Kulliyyah of Engineering, International Islamic University \\ Malaysia (IIUM), Jalan Gombak, 53100 Kuala Lumpur, Malaysia \\ ${ }^{3}$ Department of Mechatronics Engineering, Kulliyyah of Engineering, International Islamic University Malaysia (IIUM), \\ Jalan Gombak, 53100 Kuala Lumpur, Malaysia
}

\begin{tabular}{l} 
Article Info \\
\hline Article history: \\
Received May 5, 2020 \\
Revised Dec 5, 2020 \\
Accepted May 3, 2021
\end{tabular}

\section{Keywords:}

Arduino

Autonomous surface vessel

GPS

Search and rescue

Sonar sensor

\begin{abstract}
Search and rescue operation is performed to save human life, for example during natural disasters, unfortunate incidents on the land, in the deepwater, or lakes. There were incidents happened to the search and rescue crew during the operation although they were well trained. A new method using robotic technology is important to reduce the crew's risk during operations. This research proposed a development of an autonomous surface vessel for search and rescue operations for deepwater applications. The proposed autonomous surface vessel is equipped with a global positioning system (GPS) and underwater sensor to search for the victims, black box, debris, or other evidence on the surface and underwater. The vessel was designed with monitoring and control via radio frequency wireless communication. The autonomous surface vessel prototype was developed and tested successfully with the telemetry at the ground station. The ground station acts as the control centre of the overall system. Results showed the vessel successfully operated autonomously. The operator at the ground station was able to monitor the sensor data and control the vessel's manoeuvre according to the created path. The telemetry coverage to monitor the water surroundings and control the vessel's manoeuvre was around 100 meters.
\end{abstract}

This is an open access article under the CC BY-SA license.

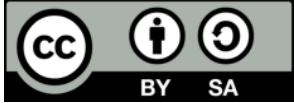

\section{Corresponding Author:}

Hasmah Mansor

Department of Electrical and Computer Engineering

International Islamic University Malaysia (IIUM)

Jalan Gombak, 53100 Kuala Lumpur, Malaysia

Email: hasmahm@iium.edu.my

\section{INTRODUCTION}

Search and rescue, SAR is an operation to search for the living victims that are in danger. However, many cases have happened where SAR crew to be in danger and involved in fatal accidents during the operation. For example, six firemen in Malaysia drown during rescuing a drowning teenager at a mine [1]. In Indonesia, during the search and rescue mission for the Lion Air aeroplane crashed, one of the divers died during the operation due to the decompression of the human body in the deep sea [2].

Nowadays, robotic technologies offer many innovative solutions to assist and reduce the risk of humans at work. Autonomous surface vessel (ASV) has been used in many areas that are considered to be dangerous and risky. Biologists have used ASV to monitor the quality of the water, the military operation has been using ASV for monitoring coastal area and detecting sea mines, drones and robots have been using in handling chemicals and other dangerous tasks those could cause health problems [3]-[5] and many more. It has been proven that the utilisation of ASV and robots have increased productivity, improved time efficiency with more flexibility. 
Recent research on ASV mainly focuses on general control objectives such as asymptotic tracking, guaranteed transient tracking performance, the stability of the vessel, robustness towards disturbances, adaptation to environmental change, and collision avoidance. Y. Hu et al. [6] has developed an avoidance system using real-time fuzzy logic, considering the steering dynamic of the ASV. Based on experience, an algorithm called fuzzy case base reasoning has been proposed. The proposed system was able to avoid a collision when similar cases happened in ASV heading control system. Z. I. Bell et al. [7] and C. Dong et al. [8] have developed adaptive controllers for ASV subject to parametric uncertainties and time-varying disturbances. The Lyapunov theory has been used as the adaptive algorithm. The vessel was successfully operated subject to parametric uncertainties and time-varying disturbances. Another challenge, ASV, when operated on the sea yaw angle, is exposed to disturbances. Therefore robust control is suggested by W. Guan et al. [9]. The technique used was closed-loop shaping, which incorporates with recursive least squares method. Astrov et al. [10] has proposed neural predictive control for ASV. The work conducted focused on modelling and simulation of a nonlinear system of ASV via Simulink/Matlab. The research conducted on ASV has successfully achieved its objectives. However, the complete design of ASV for safety and rescue mission is still not available. Therefore the design and implementation of ASV for search and rescue are proposed.

The SAR team has used a sonar sensor from Germany to find two drowned victims in the lake [11]. The mission used a side-scan sonar sensor, and it was successful. The victim was found in just several hours instead of several days for manual diver search. There were research about sidescan imaging sonar for SAR in improving the quality of sonar image. For example author in [12] proposed quantitative model for by predicting the shadow contrast ratio. With this motivation, the proposed ASV will use a sonar sensor to identify the depth of water or shape/object underwater.

SAR missions usually involved a wide coverage area. The most critical features of the ASV system's wireless communication are reliability, real-time data transfer (position and sensor status). In the HydroNet ASV water quality project, the data has been transferred using a WiFi module and 433Mhz channel module [3]. The system was able to communicate approximately $10 \mathrm{~km}$ apart between the rover and ground station and locate three rovers simultaneously during the execution of the mission. Jafaar Fahad A. Rida [13] has proposed improving mobile wireless communication based on non-line of sight; however, for this SAR application, the ASV is operated at the water surface where there are no high rise buildings and trees at the searching area. Other wireless communications that have been used in ASV are radio frequency (RF) and global system for mobile (GSM), as discussed in [14-17]. Shadman Sakib Arnob et al. [17], GSM was used to notify the control room if the passenger boat is overloaded and in danger. The author used the global positioning system (GPS) and proportional integral controller (PID) to track and avoid the autonomous boat. The proof of concept was tested on a miniature boat where the communication range of the ultrasonic sensor achieved during the experiment was between $30 \mathrm{~cm}$ to $200 \mathrm{~cm}$. Similar to SAR missions, [18] developed eCall for Malaysia's automotive industries when accidents occurred. The system automatically transmits a call to the public service answering point and certain vehicle-related information using a microcomputer, GSM, and GPS technologies when a collision was detected; to inform the emergency response unit for their immediate action.

Some examples of the application used at the ground station for ASV monitoring are XRradioLS D4 and quantum first-person view (FPV). XRLS D4 was successfully deployed with first-person view video footage and can control the rover [19]. It is costly, compared to quantum FPV. Quantum FPV ground station was built for video streaming purposes only, without control features of UAV [20]. FPV camera in a rover has also been used as visual monitoring in SAR application. From the FPV camera, information from surrounding was collected and work lifting to collect/remove load was also performed [21]. In the proposed research, the monitoring and control of ASV will be conducted by the SAR crew at the ground station where GPS data is used to determine the real-time position of ASV. All data from the ASV and sensors would be acquired.

In a more complex autonomous system such as light-detection-and-ranging (LiDAR) where various parameters are to be configured simultaneously, [22] developed genetic algorithm for robot navigation system for the robot to be navigated from one point to another in a fast and safe environment; without hitting static or dynamic obstacles. LiDAR can collect more than 1 million points per second of high-quality 3D data, make the simultaneous localisation and mapping (SLAM) algorithm for mapping and localisation using robotic operating system (ROS) important [23]. LiDAR is usually used to measure the ground's shape and contour from the air, but not suitable for underwater scanning.

In summary, ASV for search and rescue operation has been proposed in this research. The ASV is equipped with a side-scan sensor that could help in the searching operation, suitable for underwater scanning. The data collected from the sensor could be access from the ground station where the SAR team could access 
the area's current situation. The SAR team would monitor and control the ASV with the help of video footage at the ground station via RF wireless communication.

\section{RESEARCH METHOD}

The overall design of ASV for SAR proposed in this research is shown in Figure 1. It involved three components: surface vessel, ground station, and communication system. The ground station controls the ASV and monitors the monitoring aid system's data via a communication line (wireless RF). The surface vessel can be controlled by the operator remotely either in autonomous or manual mode.
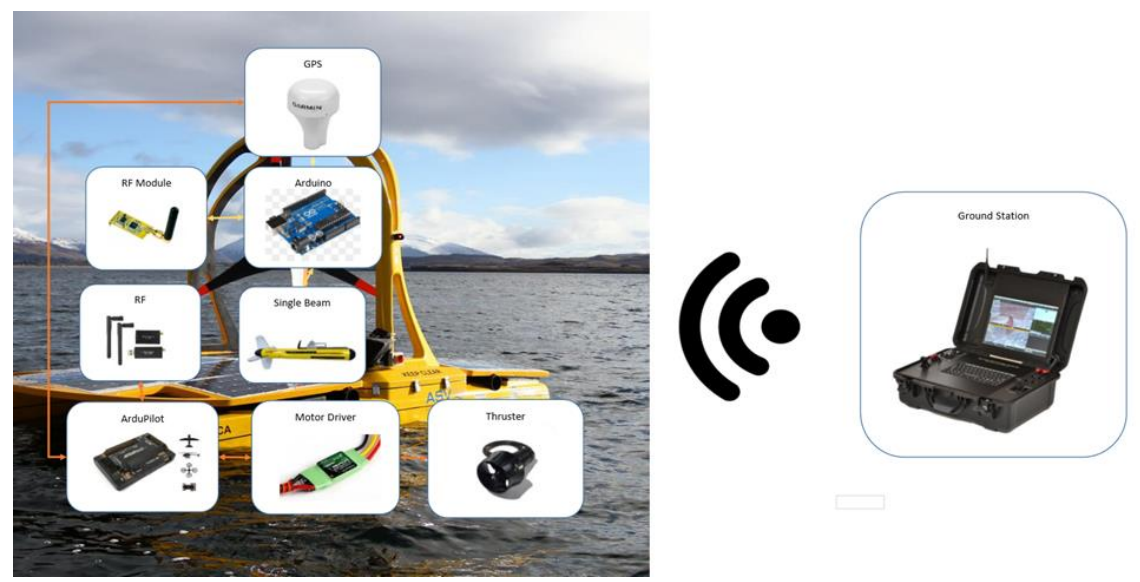

Figure 1. Overall system design of ASV for SAR

\subsection{Surface vessel}

The ASV is designed with three main components that are hull design, communication system, and manoeuvring system. The selection of hull design is essential as it affects the stability and performance of the vessel. In the hull prototype design, a single planning hull design with deep-V style has been proposed due to easy manoeuvring a higher speed and smooth ride through the rough water surface. The vessel/hull has been installed with several sensors and actuators, as shown in Table 1. All of the sensors and actuators are powered up using an $18.0 \mathrm{~V}$ LiPo battery. Two $18 \mathrm{~V}$ LiPo battery with a capacity of $5200 \mathrm{mAh}$ is connected in parallel to double output capacity to deliver to the system, thus double up the working hours.

Table 1. Electronic component specification

\begin{tabular}{|c|c|c|c|}
\hline Sensor/Actuator & Voltage (V) & Current $(\mathrm{A})$ & Supply Voltage (V) \\
\hline Servo $(\mathrm{HS}=805 \mathrm{BB})$ & $4.5-6$ & $8 \mathrm{~m}$ & 5 \\
\hline GPS (Garmin 19X HVS) & $8-33$ & $40 \mathrm{~m}$ & 18 \\
\hline Side Scan Sonar (CruzPro DSP Active Depth and Temperature Transducers) & $9.5-16$ & $3.5 \mathrm{~m}$ & 12 \\
\hline
\end{tabular}

\subsection{Portable ground station}

A portable ground system is used to monitor and control the ASV using RF telemetry. It consists of an industrial computer, wireless module connection for sensor data transfer, and remote control for manual control of the vessel manoeuvring system. LiPo battery has been chosen due to its small size and portable.

\subsection{Communication system}

In the proposed design of ASV for SAR, there are two parts of communications required. The first part is RF wireless communication used to transmit data from the side-scan sonar sensor to the ground station for underwater image analysis and perform manual control of USV. The second part is to receive data from the GPS to keep track of the ASV location and control the ASV with the desired location.

\subsubsection{Data transmission}

In the proposed system, RF wireless telemetry has been used for the communication system on data transmission due to its capability to transmit the data at long range. RF connection allows the data packet to 
be transferred between vessels and the ground station. At the ASV, the sensor data are transferred using the National Marine Electronics Association (NMEA) 0183 standard protocol. NMEA 0183 is a standard marine data protocol that provides multiple data in one input string. NMEA protocol enables one talker to provide multiple data in a single string for a listener at one time. Besides that, the NMEA 0183 standard data protocol provides extra reliability on data transmission [24]. In this system, the sensors that are using NMEA protocol are GPS and single beam side scan sonar. Since more than one sensor is used in this system, the data from the sensors are combined using the NMEA data combiner to make the data transfer across one telemetry connection. The architecture of the communication system is shown in Figure 2.

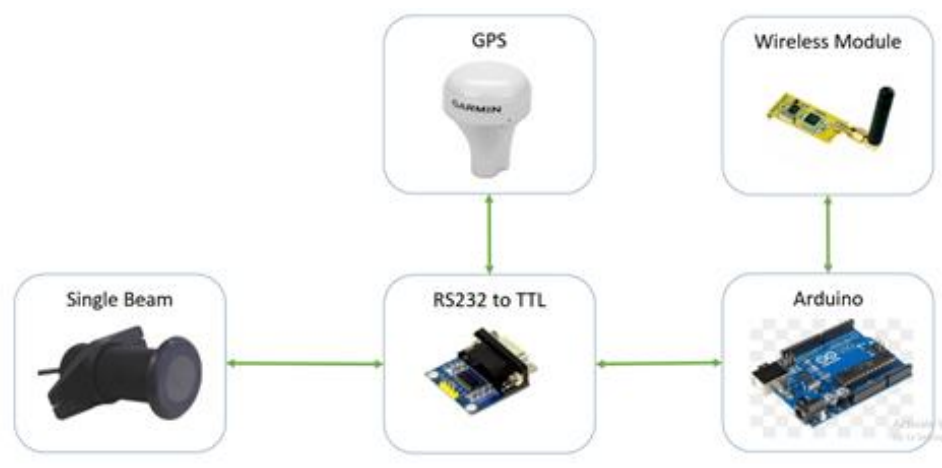

Figure 2. The architecture of the ASV communication system

\subsubsection{Autonomous system}

In Figure 3, the ASV has been set with the desired location in the autonomous system design. A microcontroller, ArduPilot Mega (APM), has been used and installed at the vessel. The APM has been programmed to operate the vessel autonomously from the ground station.

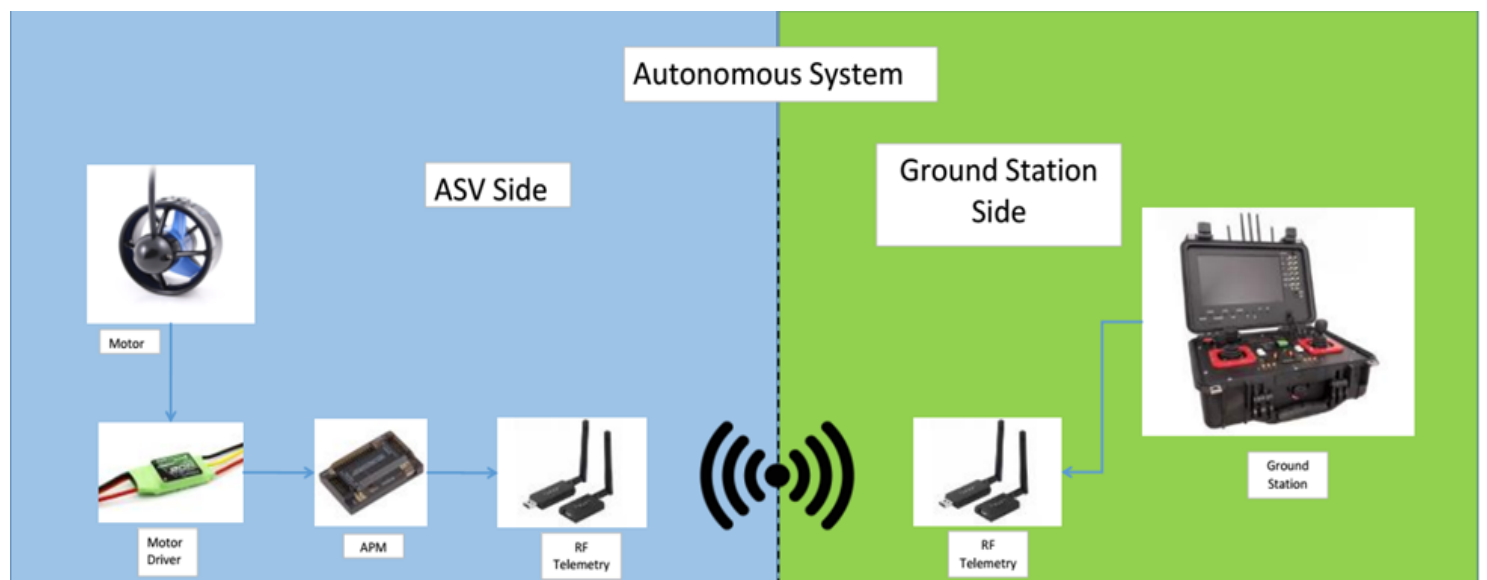

Figure 3. Autonomous system diagram

The ASV could be monitored and controlled from the ground station wirelessly. The connection has been established using RF telemetry communication between the ground stations with the vessel. Data of the specific setting and desired location have been sent from the ground station to the microcontroller. The microcontroller has processed all the data by comparing it with the GPS and inertial measurement unit (IMU) data. Based on the comparison between these data, the APM control the vessel autonomously.

\subsubsection{Search and rescue monitoring system}

Search and rescue monitoring system has been set at the ground station. Data from side-scan sonar and global positioning system, GPS are received. Side-scan sonar provides surrounding data on the water surface and underwater and draws the underwater image that made underwater searching possible. GPS sensor is used to provide location data of the vessel. 


\section{RESULTS AND DISCUSSION}

The integration of the overall system, as shown in Figure 4, has been done to evaluate the ASV system's performance. GPS and single beam echo sounder (sonar sensor) have been installed at the vessel, the communication system and the ground station have been integrated. The system was tested at Tasek Selayang, Malaysia.

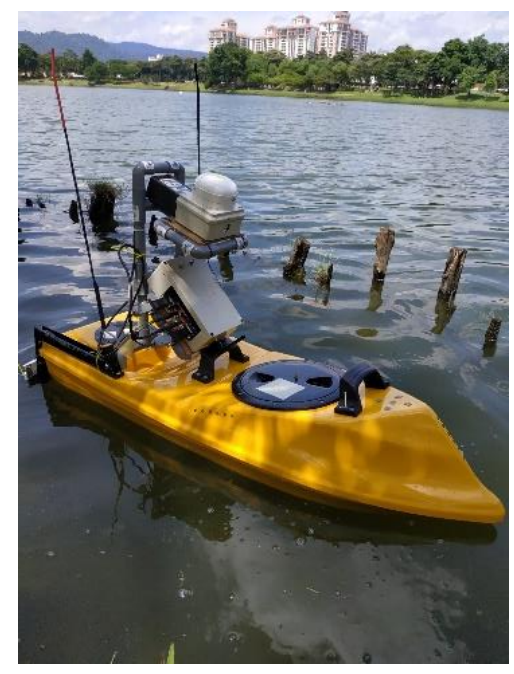

Figure 4. Experimental setup of ASV

\subsection{Experiment on global positioning sensor data}

In this project, GPS has been used to provide real-time positioning of the vessel. The sensor used an RS232 serial communication interface connected to the computer or sensor. In order to read the data of the sensor, the GPS was connected to the computer. Data was read at the computer, using raw data reader software called Teraterm. Figure 5(a) shows the data of the GPS using the NMEA 0183 standard protocol. This data can be used in mapping software to visualise the real-time position of the vessel. The definition of each NMEA sentence identifier can be found in [25].

\subsection{Experiment on single beam echo sounder data}

In this research, a sonar sensor was used to help in underwater search operations by scanning an underwater image. Figure 5(b) shows single beam echo sounder NMEA 0183 data sentences obtained from the experimental work at Tasek Selayang. The data contained information on the depth and temperature of the water.

\subsection{Experiment on wireless serial communication}

All the sensors' data from the surface vessel was sent wirelessly to the ground station for monitoring purposes. The Arduino read the data and then sent to the ground station through the HC-12 wireless module. Figure 6(a) shows the developed wireless communication prototype of the overall autonomous system diagram (ASV side) of Figure 4. The sensor used RS232 physical protocol, which used 12V signal logic. Due to the ArduPilot Mega (APM) microcontroller signal requirement of $\pm 5 \mathrm{~V}, \mathrm{RS} 232$ to TTL converter was used to convert $12 \mathrm{~V}$ signal logic to $5 \mathrm{~V}$ signal logic. APM then sent the sensor data via the HC-12 transceiver wireless module to the ground station. The data was received at the ground station through the HC-12 transceiver module, which was connected to another Arduino microcontroller. The Arduino was connected to the ground station's computer via a USB port. In order to check the connection, the Teraterm software was used to display all the data received from the vessel to the ground station.

\subsection{Experiment on real-time positioning}

Using google earth pro software, the real-time GPS data were plotted during the experimental test at Tasek Selayang. The result showed that the location of the vessel was plotted and display at the ground station. As the vessel moved across the lake, its route was mapped on the Google Earth Pro as a red line. The result is shown in Figure 6(b). The data of the GPS were sent by APM using the HC-12 wireless module. At the ground station, the receiver was connected to the computer and displayed the vessel movement's position using google earth pro software. 
\$GPRMC , 053645. 3, A, 0315.17402, N, 10143.82493, E, 000.17, 356.5, 171018,000.2,W, A* \$GPGGA, 053645.3,0315.17402, N, 10143.82493, E, 1, 08,1.0, 80.6, M, - 3.6, M, , *75 \$GPVTG, 356.5, T, 356.6, M, 000.17, N, 0000.31, K, A*14

\$GPRMC, 053645.4, A, 0315.17402, N, 10143.82493, E, 000.19, 356.5, 171018,000.2,W, A* \$GPGGA, 053645.4,0315.17402, N, 10143.82493, E, 1, 08,1.0, 80.6, M, - 3.6, M, , *72 \$GPVTG, 356.5, T, 356.6, M, 000.19, N, 0000.35, K, A*1E

\$GPRMC, 053645.5, A, 0315.17402, N, 10143.82493, E, 000.19, 356.5, 171018,000.2, W, A* \$GPGGA, 053645.5, 0315.17402, N, 10143.82493, E, 1, 08, 1.0, 80.6, M, - 3.6, M, , *73 $\$$ GPVTG, 356.5, T , 356.6, M, 000.19, N, 0000.35, K, A*1E

\$GPRMC, 053645.6, A, 0315.17402, N, 10143.82493, E, 000.19, 356.5, 171018, 000.2, W, A* \$GPGGA, 053645.6,0315.17402, N, 10143.82493, E, 1, 08,1.0, 80.6, M, - 3.6, M, , *70 \$GPVTG, 356.5, T, 356.6, M, 000.19, N, 0000.35, K, A*1E

\$GPRMC, 053645.7, A, 0315.17402, N, 10143.82493, E, 000.21, 356.5, 171018,000.2, W, A* \$GPGGA, 053645.7,0315.17402, N, 10143.82493, E, 1, 08,1.0, 80.6, M, - 3.6, M, , *71 \$GPVTG, 356.5, T, 356.6, M, 000.21, N, 0000. 38, K, A*18

\$GPRMC, 053645. 8, A, 0315.17402, N, 10143.82493, E, 000.21, 356.5, 171018,000.2, W, A* \$GPGGA, 053645.8,0315.17402, N, 10143.82493, E, 1, 08,1.0, 80.6, M, - 3.6, M, , *7E \$GPVTG, 356.5, T, 356.6, M, 000.21, N, 0000.38, K, A*18

\$GPRMC, 053645.9, A, 0315.17396, N, 10143.82493, E, 000.24, 356.5, 171018, 000.2, W, A* \$GPGGA, 053645.9,0315.17396, N, 10143.82493, E,1,08,1.0,80.6, M, -3.6, M, , *75 \$GPVTG, 356.5, T, 356. 6, M, 000. 24, N, 0000.44, K, A*16
\$SDMTW, 033.8, C*3C \$SDDPT, $002.6, * 7 \mathrm{D}$

\$SDDBT, $008.5, f, 002.6, M, 001.4, F^{*} 0 A$ \$SDMTW, $033.8, \mathrm{C} * 3 \mathrm{C}$ \$SDDPT, $002.6, * 7 D$

\$SDDBT, $008.5, f, 002.6, M, 001.4, F^{*} 0 A$ \$SDMTW, $033.8, \mathrm{C} * 3 \mathrm{C}$

\$SDDPT, $002.6, * 7 D$

\$SDDBT, $008.5, \mathrm{f}, 002.6, \mathrm{M}, 001.4, \mathrm{~F} * 0 \mathrm{~A}$

\$SDMTW, $033.8, \mathrm{C} * 3 \mathrm{C}$

\$SDDPT, $002.6, * 7 D$

\$SDDBT, $008.5, \mathrm{f}, 002.6, \mathrm{M}, 001.4, \mathrm{~F} * 0 \mathrm{~A}$

\$SDMTW, $033.8, \mathrm{C} * 3 \mathrm{C}$

\$SDDPT, 002.6,*7D

$\$ S D D B T, 008.5, \mathrm{f}, 002.6, \mathrm{M}, 001.4, \mathrm{~F}^{*} 0 \mathrm{~A}$

\$SDMTW, $033.8, \mathrm{C} * 3 \mathrm{C}$

$\$ S D D P T, 002.6, * 7 D$

(a)

(b)

Figure 5. These figures are, (a) Example of GPS, (b) Single beam echo sound raw data

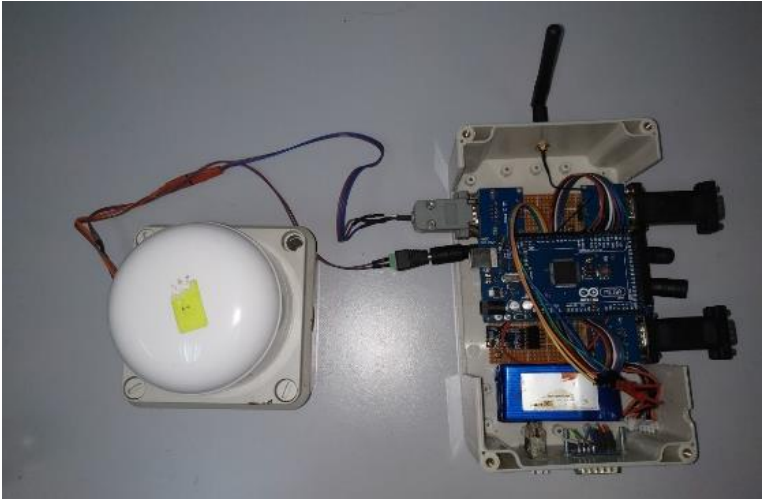

(a)

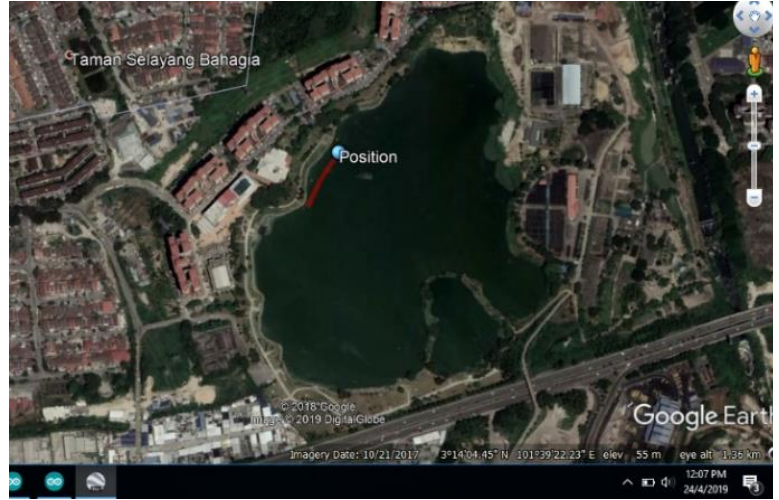

(b)

Figure 6. Wireless module and path movement, (a) Wireless communication module, (b) Vessel path movement on google earth pro

\subsection{Experiment on single beam echo sounder underwater mapping}

A single beam echo sounder was used to scan the 2D image of the underwater cross-section where the depth and shape underwater surface could be identified. The sensor data was sent through the wireless module and received by the Arduino at the ground station in real-time. Since there is no available open-source software to sketch the 2D underwater image, NMEA data was used by the microcontroller to obtain the depth of water. The data was visualised using a serial plotter function in Arduino IDE, as shown in Figure 7. From the graph, the depth of the lake water is around 5 meters.

\subsection{Experiment on the autonomous system}

In the autonomous system, the Ardupilot board or APM was used to control the vessel's movement using open-source software, Mission Planner. The board was placed on the vessel and controlled from the ground station using a telemetry receiver that receives the flight data from the telemetry transmitter at the ground station. The vessel was controlled using the path planning function, which guides the vessel's movement according to the path created.

The autonomous system was tested on the lake. First, the path was created using the Mission Planner. Then, the path was uploaded to the vessel via telemetry (transmitter). Once the telemetry (receiver) received the signal, the vessel started the mission by following the path created. As shown in Figure 8 , the path created is shown in the yellow line. The purple line shows the actual direction of the vessel. Due to some factors from the climate change during the experiment, the telemetry coverage to monitor the underwater image and control the vessel's manoeuvre was around 100 meters. 


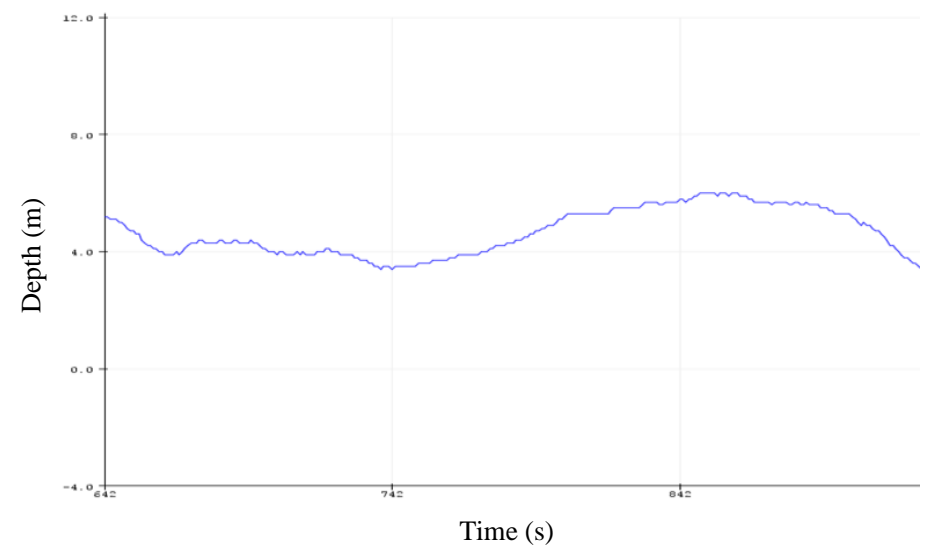

Figure 7. Graph showing the depth of the water

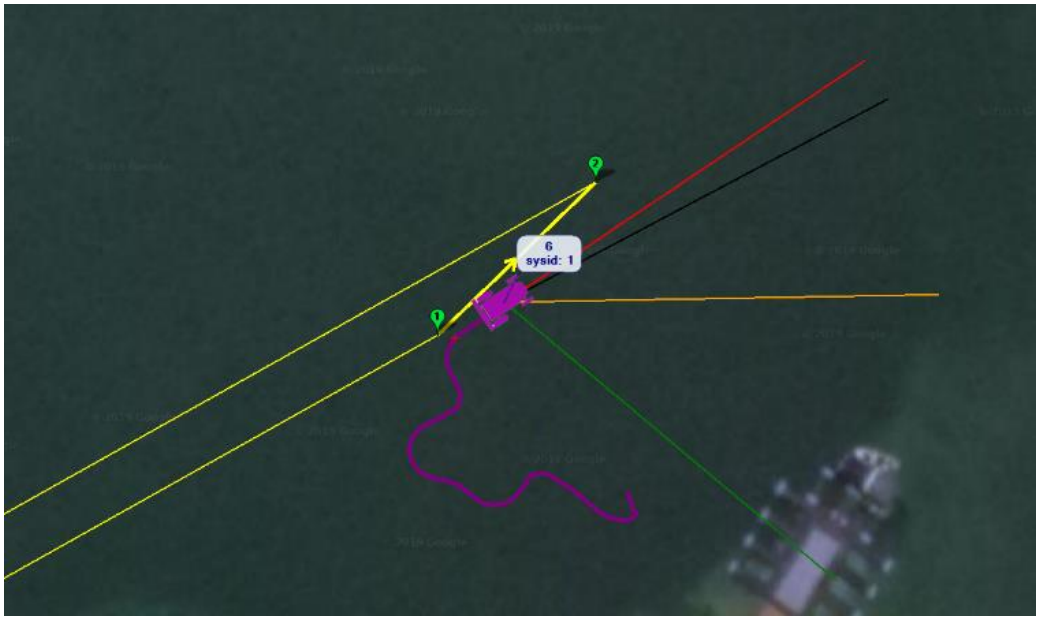

Figure 8. Experiment on path planning of the autonomous system

\section{CONCLUSION}

In conclusion, the objective of this research has been achieved. An autonomous surface vessel for search and rescue for a deepwater operation has been designed and developed. The developed prototype includes a portable ground station where RF wireless communication has used the communication between the vessel and the ground station. Experiment results showed that the proposed system could scan the 2D image of the underwater cross-section, which were the depth and temperature of the water; shown at the ground station. When the SAR crew created a path at the telemetry, the vessel moved successfully to the created path. Future research includes integration with more sensors for intelligence, surveillance, command and control for the possibility to be implemented for military purposes.

\section{ACKNOWLEDGMENTS}

The authors would like to thank Centre of Unmanned Technology (CUTe) and International Islamic University Malaysia (IIUM) for providing the research and development facility and the full support towards the completion of this project.

\section{REFERENCES}

[1] The chronology of tragedy 6 members of Bomba Limp in Bahasa. [Online]. Available: https://www.bharian.com.my/berita/kes/2018/10/481651/kronologi-tragedi-6-anggota-bomba-lemas. [Access: April $1,2020]$

[2] Lion JT610: A diver died during the evacuation process in Bahasa. [Online]. Available: https://www.bbc.com/indonesia/indonesia-46081626. [Access: April 15, 2020]

[3] G. Ferri, A. Manzi, F. Fornai, F. Ciuchi and C. Laschi, "The HydroNet ASV, a Small-Sized Autonomous Catamaran for Real-Time Monitoring of Water Quality: From Design to Missions at Sea," in IEEE Journal of Oceanic Engineering, vol. 40, no. 3, pp. 710-726, July 2015, doi: 10.1109/JOE.2014.2359361. 
[4] M. A. M. Basri and A. Nordin, "Optimal Backstepping Control of Quadrotor UAV using Gravitational Search Optimization Algorithm," in Bulletin of Electrical Engineering and Informatics, vol. 9, no. 5, pp. 1819-1826, 2020.

[5] A. Benedetto, P. Luca, M. Marco, A. Corrieri, D. Masti and L. Vanni, "Development of an innovative and sustainable sail-drone," 2017 IEEE International Conference on Environment and Electrical Engineering and 2017 IEEE Industrial and Commercial Power Systems Europe (EEEIC /I\&CPS Europe), pp. 1-6, 2017.

[6] Y. Hu, X. Meng, Q. Zhang and G. Park, "A Real-Time Collision Avoidance System for Autonomous Surface Vessel Using Fuzzy Logic," in IEEE Access, vol. 8, pp. 108835-108846, 2020, doi: 10.1109/ACCESS.2020.3001626.

[7] Z. I. Bell, J. Nezvadovitz, A. Parikh, E. M. Schwartz and W. E. Dixon, "Global Exponential Tracking Control for an Autonomous Surface Vessel: An Integral Concurrent Learning Approach," in IEEE Journal of Oceanic Engineering, vol. 45, no. 2, pp. 362-370, April 2020, doi: 10.1109/JOE.2018.2880622.

[8] C. Dong, S. He and S. -L. Dai, "Performance-Guaranteed Tracking Control of an Autonomous Surface Vessel With Parametric Uncertainties and Time-Varying Disturbances," in IEEE Access, vol. 7, pp. 101905-101914, 2019, doi: 10.1109/ACCESS.2019.2931337.

[9] W. Guan, W. Cao, J. Sun and Z. Su, "Steering Controller Design for Smart Autonomous Surface Vessel Based on CSF L2 Gain Robust Strategy," in IEEE Access, vol. 7, pp. 109982-109989, 2019, doi: 10.1109/ACCESS.2019.2931887.

[10] I. Astrov, A. Udal, I. Roasto and H. Mõlder, "Target Tracking by Neural Predictive Control of Autonomous Surface Vessel for Environment Monitoring and Cargo Transportation Applications," 2020 17th Biennial Baltic Electronics Conference (BEC), 2020, pp. 1-4, doi: 10.1109/BEC49624.2020.9277115.

[11] Side-scan sonar for first responders \& search and rescue (SAR). [Online]. Available: https://blacklaserlearning.com/sonar-for-first-responders-marine-rescue/. [Access: April 3, 2020]

[12] D. A. Cook and D. C. Brown, "Synthetic Aperture Sonar Image Contrast Prediction," in IEEE Journal of Oceanic Engineering, vol. 43, no. 2, pp. 523-535, 2018.

[13] Jafaar Fahad A. Rida, "Improvement for performance radio frequency in wireless communication based on impulse signal," Indonesian Journal of Electrical Engineering \& Computer Science (IJEECS), vol 18, no 2, pp. $903-916$ May 2020, doi: 10.11591/ijeecs.v18.i2.pp903-916.

[14] A. Denker and M. C. İşeri, "Design and implementation of a semi-autonomous mobile search and rescue robot: SALVOR," 2017 International Artificial Intelligence and Data Processing Symposium (IDAP), 2017, pp. 1-6, doi: 10.1109/IDAP.2017.8090184.

[15] Hui Liu, Shuaiqi Gan, Jialei Zhang and Xianbo Xiang, "Control system of a mini-ASV prototype: Design and implementation," 2016 IEEE International Conference on Underwater System Technology: Theory and Applications (USYS), Penang, 2016, pp. 110-114.

[16] Z. Z. Abidin, M. R. Arshad and U. K. Ngah, "Waypoint control of Drosobots: Swarms of mini ASVs," 2010 IEEE Symposium on Industrial Electronics and Applications (ISIEA), Penang, Malaysia, 2010, pp. 556-561, doi: 10.1109/ISIEA.2010.5679401.

[17] Shadman Sakib Arnob, Adiba Sumaiya Khan, Rashed Shelim, Mahmood Chowdhury, "Safe sailing: GSM and GPS controlled autonomous boat with overweight detection and obstacle avoidance," Indonesian Journal of Electrical Engineering \& Computer Science (IJEECS), vol. 14, no. 2, pp. 715-724, May 2019, doi: 10.11591/ijeecs.v14.i2.pp715-724.

[18] M. A. Mushthalib, H. Mansor and Z. Z. Abidin, "Development of eCall for Malaysia's Automotive Industries," 2019 7th International Conference on Mechatronics Engineering (ICOM), Putrajaya, Malaysia, 2019, pp. 1-5, doi: 10.1109/ICOM47790.2019.8952044.

[19] XLRS D4: Ground Control Station PFV \& UAV. [Online]. Available: https://d3.xlrs.eu/en/xlrs_d4/. [Access: Jun 12, 2020].

[20] Quantum FPV Ground Station Review. [Online]. Available: http://www.droneuplift.com/quanum-fpv-groundstation-review/. [Access: Jun 12, 2020]

[21] R. M. Ramli, N. A. Herman, M. R. Mazlan, A. S. Nazaruddin, M. A. Tuah, A. N. E Abd S, K Othman, Y. M Esmail "Development of Robotic Rover with Controller \& Vision System," Indonesian Journal of Electrical Engineering \& Computer Science (IJEECS), vol. 18, no. 2, pp. 766-773, May 2020 doi: 10.11591/ijeecs.v18.i2.pp766-773.

[22] S. N. Anual, M. F. Ibrahim, N. Ibrahim, A. Hussain, M. M. Mustafa, A. Baseri Huddin, F. H. Hashim, "GA-based Optimisation of a LiDAR Feedback Autonomous Mobile Robot Navigation System," Bulletin of Electrical and Informatics (BEEI), vol. 7, no 3, pp. 433-441, September 2018, doi: 10.11591/eei.v7i3.1275.

[23] S. Abdul-Rahman, M. S. Abd Razak, A. H. Mohd Mushin, R. Hamzah, N. Abu Bakar, and Z. Abd Aziz. "Simulation of simultaneous localisation and mapping using point cloud data," Indonesian Journal of Electrical Engineering and Computer Science (IJEECS), vol. 16, no 2, pp. 941-949, 2019, doi: 10.11591/ijeecs.v16.i2.pp941949.

[24] R. Coleman, "A Self-Healing Plug-in Parser for NMEA Streams," Fifth International Conference on Information Technology: New Generations (itng 2008), 2008, pp. 1023-1027, doi: 10.1109/ITNG.2008.238.

[25] M. Shoab, K. Jain, M. Anulhaq and M. Shashi, "Development and implementation of NMEA interpreter for real time GPS data logging," 2013 3rd IEEE International Advance Computing Conference (IACC), Ghaziabad, India, pp. 143-146, 2013. 